\title{
Potência muscular e capacidade de sprints repetidos em jogadores de futebol
}

\author{
Muscle power and repeated sprint ability in soccer players
}

\author{
Juliano Dal Pupo \\ Carlos Miguel Porto Almeida \\ Daniele Detanico \\ Juliano Fernandes da Silva ${ }^{2}$ \\ Luiz Guilherme Antonacci Guglielmo \\ Saray Giovana dos Santos 1
}

1 Universidade Federal de Santa Catarina. Laboratório de Biomecânica, Programa de Pós-Graduação em Educação Física. Florianópolis, SC. Brasil;

2 Universidade Federal de Santa Catarina. Laboratório de Esforço Físico, Programa de PósGraduação em Educação Física. Florianópolis, SC. Brasil.

Recebido em 11/11/09 Revisado em 16/12/09 Aprovado em 08/01/10
Resumo - A potência muscular é uma das qualidades físicas mais importantes da perfor mance de jogadores de futebol, sendo imprescindível a manutenção da mesma no decorrer da partida. Assim, objetivou-se investigar os níveis de potência muscular em jogadores de futebol antes e após realizarem sprints repetidos (SR), assim como as relações entre a potência e o desempenho nos SR. Participaram 20 jogadores de futebol da categoria sub-20, com idades entre 18-20 anos. O design do estudo consistiu em: execução dos saltos verticais; execução dos SR; nova execução de saltos verticais e coleta de amostra sanguínea. Para a mensuração da potência muscular realizou-se o Continuous Jump (CJ) sobre uma plataforma de força piezoelétrica e para a avaliação dos SR, foi utilizado o RAST test. Não foi observada diferença significativa nos níveis de potência muscular após a realização dos SR ( $p=0,57)$. Verificou-se diferença significativa nos tempos do primeiro até o quinto sprint $(\mathrm{p}<0,01)$, não sendo observada diferença entre o quinto e o sexto $(p=0,06)$. A altura no CJ antes da realização dos SR correlacionou-se com o tempo no primeiro sprint $(\mathrm{r}=-0,62 ; \mathrm{p}<0,01)$, com o melhor tempo $(\mathrm{r}=-0,60 ; \mathrm{p}<0,01)$ e com o tempo médio nos sprints $(\mathrm{r}=-0,54 ; \mathrm{p}<0,01)$. Podese concluir que os jogadores analisados não apresentaram redução significativa nos níveis de potência após a realização dos SR. Houve decréscimo do desempenho do primeiro até o quinto sprint, sendo mantido entre o quinto e o sexto. Os níveis de potência muscular foram determinantes na capacidade dos jogadores realizarem um sprint máximo, assim como sucessivos sprints.

Palavras-chave: Força muscular; Fadiga muscular; Futebol.

Abstract - Muscle power is one of the most important physical qualities of soccer player performance and needs to be maintained during a match. Thus, the aim of this study was to investigate the levels of muscle power in soccer players before and after performing repeated sprints (RS), and the association between power and RS performance. Twenty soccer players from the under-20 category aged 18-20 years participated in this study. The study consisted of the execution of vertical jumps, execution of RS, new execution of vertical jumps, and collection of blood samples. The continuous jump (CJ) test was performed on a piezoelectric force platform for the measurement of muscle power and the RAST test was used to evaluate RS ability. No significant difference in the levels of muscle power was observed after RS ( $p=0.57)$. Significant differences were observed in the first to fifth sprint times $(p<0.01)$, but not between the fifth and sixth sprint $(p=0.06)$. CJ height before $R S$ was correlated with first sprint time $(r=-0.62, p<0.01)$, best sprint time $(r=-0.60, p<0.01)$, and average sprint time $(r=-0.54, p<0.01)$. In conclusion, the soccer players studied showed no significant reduction in muscle power after RS. A decrease in performance was observed from the first to the fifth sprint, but not between the fifth and sixth sprint. The muscle power of soccer players was a determinant factor to perform one maximum sprint, as well as successive sprints.

Key words: Muscle strength; Muscle fatigue; Soccer. 


\section{INTRODUÇÃO}

O futebol pode ser considerado uma modalidade esportiva na qual solicita dos jogadores, além de condições técnicas e táticas, diferentes qualidades físicas. Dentre estas destaca-se a potência muscular, caracterizada como a taxa de realização de trabalho em determinado período, mais especificamente, o produto da força pela velocidade ${ }^{1}$, a qual pode ser considerada uma das variáveis determinantes da performance de jogadores de futebol, pois está relacionada com a maioria das ações de jogo, tais como os chutes, os saltos para o cabeceio, dentre outras ${ }^{2}$.

Uma das ações determinantes durante uma partida de futebol é a realização de deslocamentos curtos em intensidades máximas ou quase máximas, intercaladas com breves períodos de recuperação ao longo da partida, denominados de sprints. Estes estímulos costumam representar apenas $1-3 \%$ do tempo total de jogo ${ }^{3}$, porém são nestes momentos que ocorrem as ações decisivas. A capacidade de realizar sucessivos sprints na maior velocidade possível é denominada capacidade de sprints repetidos $(\mathrm{CSR})^{4}$, que tem sido considerada como um dos principais componentes da aptidão física do jogador de futebol ${ }^{5-7}$.

De acordo com investigações, a potência muscular, estimada pelo desempenho obtido no Squat Jump (SJ), está correlacionada com o tempo em sprints curtos $(5 \mathrm{~m})^{8}$, assim como em distâncias maiores (10 e $30 \mathrm{~m})^{2}$. Em outro estudo ${ }^{9}$, foi reportada correlação entre o desempenho em sprints de $30 \mathrm{~m}$ e a altura no Counter Movement Jump (CMJ). Tais dados indicam a que a potência muscular associada à capacidade de recrutamento neural e aproveitamento de energia elástica são fatores que podem ser determinantes na realização destas ações de jogo.

A realização de sucessivos sprints e demais ações de alta intensidade, durante uma partida de futebol, são mantidas, inicialmente, pelos fosfatos de alta energia ${ }^{10}$. Tem sido demonstrado que a realização de vários estímulos com períodos curtos de recuperação não são suficientes para a restauração do estoque de fosfocreatina, ocorrendo, consequentemente, um aumento da participação da via glicolítica para atender a demanda metabólica ${ }^{10}$, o que explica, em parte, a redução da CSR no decorrer do tempo 5 .

Contudo, ao mesmo tempo em que há um aumento da produção energética pelo metabolismo glicolítico (lático), ocorre maior formação de íons $\mathrm{H}^{+}$, aumentando a acidez muscular, o que é considerado um fator inibidor das enzimas glicolíticas, vindo a limitar a atividade desta via ${ }^{11}$. Além disso, é reco- nhecido que o aumento dos íons $\mathrm{H}^{+}$provoca uma redução na capacidade contrátil do músculo, induzindo a uma possível fadiga intramuscular ${ }^{12}$, podendo comprometer o mecanismo da contração muscular ${ }^{13}$ e, consequentemente, a produção de potência. Diante disso, a capacidade de tamponamento ácido-base é considerada um importante atributo para manter a performance durantes estes esforços 5 .

Considerando que a manutenção de níveis ótimos de potência e a recuperação rápida entre ações intensas são imprescindíveis para o bom desempenho do futebolista, torna-se importante a análise dos efeitos agudos de uma sucessão de estímulos de alta intensidade (sprints repetidos - SR) sobre a capacidade de gerar potência muscular.

Diante disto e tendo em vista que não foram encontrados estudos que investigassem tais aspectos, a presente investigação objetivou comparar a potência de membros inferiores obtida antes e depois de uma sequência de sprints e relacionar a performance nestes com o nível de potência muscular dos jogadores. Formulou-se como uma hipótese deste estudo que os níveis de potência diminuiriam após a realização dos SR.

\section{PROCEDIMENTOS METODOLÓGICOS}

\section{Caracterização do estudo}

Este estudo pode ser classificado como pré-experimental, no qual consideraram-se os níveis de potência muscular como variável dependente e os SR como variável independente. Foram controlados fatores como a idade, o tempo de prática e o intervalo entre a realização dos SR e o teste para avaliação da potência.

\section{Sujeitos}

Participaram deste estudo, 20 jogadores de futebol da categoria sub-20, com idades entre 18 e 20 anos, pertencentes a um clube de futebol profissional participante da Série "A" do Campeonato Brasileiro. Os jogadores apresentaram as seguintes características: massa corporal $=75,89 \pm 6,6 \mathrm{~kg}$; estatura $=1,79$ $\pm 0,08 \mathrm{~m}$; gordura corporal $=10,12 \pm 2,34 \%$.

\section{Instrumentos e procedimentos}

Antes de iniciarem os procedimentos para a coleta de dados, os atletas que participaram do estudo foram esclarecidos sobre os objetivos e os métodos da pesquisa, para então assinarem o Termo de Consentimento Livre e Esclarecido. O presente estudo foi aprovado pelo Comitê de Ética em Pesquisas com Seres Humanos da UFSC (073/2007). 
Antecedendo as coletas de dados, os jogadores realizaram aquecimento prévio, que foi constituído em 5 min de corrida a baixa intensidade (trote), seguido por 3 exercícios de corrida progressiva (aproximadamente $30 \mathrm{~m}$ ). Posteriormente, as coletas foram realizadas com o seguinte design: 1) execução dos saltos verticais; 2) execução dos sprints e; 3) nova execução de saltos verticais e coleta de amostra sanguínea.

Protocolo de avaliação da potência muscular O nível de potência muscular dos jogadores foi avaliado a partir do desempenho obtido (altura) nos saltos verticais denominados Continuous Jump $(\mathrm{CJ})^{14}$. O protocolo do CJ consiste em partir de uma posição em pé, com as mãos na cintura e executar os saltos com contra-movimento, flexionando os joelhos até próximo a $90^{\circ}$. Utilizou-se como instrumento de medida da altura de salto uma plataforma de força portátil, do tipo piezoelétrica (Quattro Jump, KISTLER ${ }^{\circledR}$, modelo 9290AD), que mensura a força vertical. A fim de verificar o efeito dos sprints repetidos na potência muscular, o CJ foi realizado em dois momentos: antes e 1,5 min após a realização do Rast Test. Foram realizados três saltos contínuos, utilizando-se a média da altura como indicador dos níveis de potência muscular.

Avaliação da capacidade de sprints repetidos (CSR)

Para avaliar a CSR, foi utilizado o RAST test (Running-based Anaerobic Sprint Test) proposto por Zacharogiannis et al. ${ }^{15}$, sendo, recentemente, apresentado sua validade ${ }^{14}$. O RAST consiste em realizar 6 sprints máximos de $35 \mathrm{~m}$, em um campo de futebol, com intervalo de $10 \mathrm{~s}$ de pausa passiva entre os sprints. Na realização do teste, foram utilizadas duas fotocélulas eletrônicas Speed Test 4.0 (CEFISE), dispostas à $35 \mathrm{~m}$ de distância, para capturar os tempos dos sprints. Para fins de análise, utilizaram-se os tempos adquiridos em cada sprint (T1, T2, T3, T4, T5 e T6), o melhor tempo (MT) e o tempo médio (TM). Além disso, calculou-se um índice de fadiga (IF), de acordo com a seguinte equação:

$$
\mathrm{IF}=\left[\left(\sum \text { tempos } / 6^{*} \mathrm{MT}\right)-1\right]^{*} 100
$$

onde $\mathrm{MT}=$ melhor tempo.

Análise do lactato sanguíneo

A fim de estimar a participação da via glicolítica na realização do Rast Test, foram coletadas amostras de $25 \mu \mathrm{L}$ de sangue do lóbulo da orelha com um capilar heparinizado no $5^{\circ}$ minuto de recuperação.
O sangue foi armazenado em microtúbulos de polietileno com tampa (tipo eppendorff), sendo, posteriormente, realizada a leitura da concentração de lactato sanguíneo em um analisador eletroquímico YSI 2700 (modelo STAT SELECT).

\section{Análise estatística}

Para análise dos dados, foi utilizada estatística descritiva (média e desvio-padrão), teste de ShapiroWilk para verificar a normalidade, teste " $t$ " de Student para amostras dependentes para comparar os níveis de potência antes e após os sprints, análise de variância para medidas repetidas, seguida pelo teste de Bonferroni para comparar os tempos dos sprints e correlação linear de Pearson para relacionar os níveis de potência com os sprints. Adotou-se um nível de significância com $\mathrm{p} \leq 0,05$.

\section{RESULTADOS}

Na figura 1, está apresentada a comparação do nível de potência muscular, mensurada por meio do desempenho obtido no CJ (altura de salto), em dois momentos: antes $(42,74 \pm 3,35 \mathrm{~cm})$ e depois $(42,43 \pm 2,81 \mathrm{~cm})$ da realização dos sprints repetidos no RAST test. Não foram observadas diferenças significativas $(p=0,57)$ entre as duas situações, rejeitando assim a hipótese deste estudo.

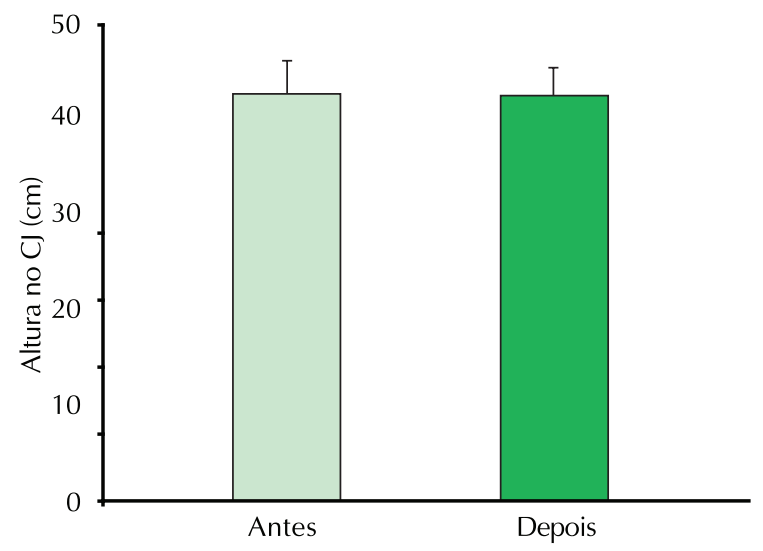

Figura 1 - Comparação da altura de salto obtida antes e depois da realização de sprints repetidos.

Quando comparados os tempos dos 6 sprints obtidos no RAST test, verificou-se diferença significativa $(\mathrm{F}=122,8 ; \mathrm{p}<0,01)$ entre os mesmos $(\mathrm{T} 1=$ $4,98 \pm 0,20 \mathrm{~s} ; \mathrm{T} 2=5,06 \pm 0,22 \mathrm{~s} ; \mathrm{T} 3=5,26 \pm 0,25 \mathrm{~s} ;$ $\mathrm{T} 4=5,36 \pm 0,22 \mathrm{~s} ; \mathrm{T} 5=5,55 \pm 0,27 \mathrm{~s}$ e $\mathrm{T} 6=5,60$ $\pm 0,29 \mathrm{~s})$, com exceção entre T5 e T6 ( $\mathrm{p}=0,06)$, conforme apresentado na figura 2 . O tempo médio nos sprints (TM) foi de 5,30 $\pm 0,23 \mathrm{~s}$ e o melhor tempo (MT) foi de 4,97 $\pm 0,21 \mathrm{~s}$.

O índice de fadiga calculado a partir do desempenho no teste foi de $6,79 \pm 1,41 \%$. A concentração 
de lactato sanguíneo obtida nos jogadores, cinco minutos após a realização do RAST test, apresentou valores médios de 10,12 $\pm 1,48$ mmol.L-1. .

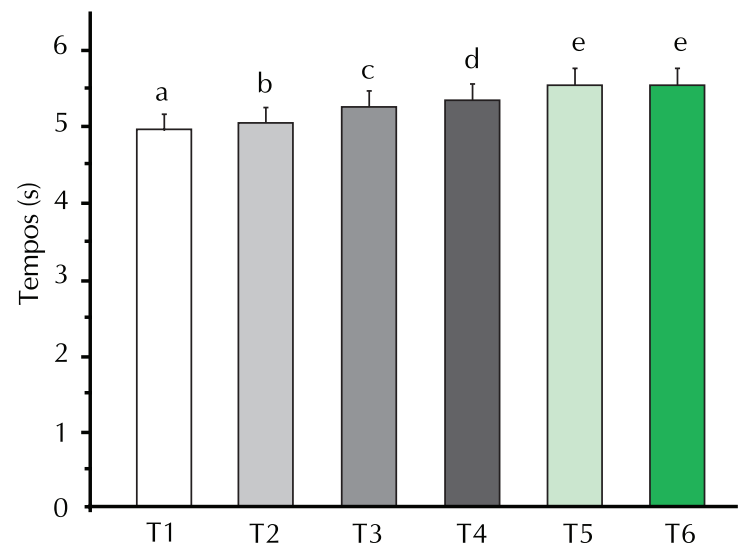

Figura 2 - Comparação entre o tempo obtido nos seis sprints (T1, T2, T3, T4, T5 e T6). (Nota: letras diferentes mostram diferença significativa).

Conforme apresentado nas figuras a seguir, observaram-se correlações negativas entre o desempenho no CJ (altura do salto) antes da realização dos sprints (Ha) com o desempenho no primeiro sprint (T1) (figura 3), entre Ha e o tempo médio dos sprints (TM) (figura 4) e entre Ha com o melhor tempo (MT) (figura 5). Porém, entre a altura depois (Hd) e o desempenho no sexto sprint (T6) não se encontrou correlação significativa $(r=-0,43 ; p=0,06)$.

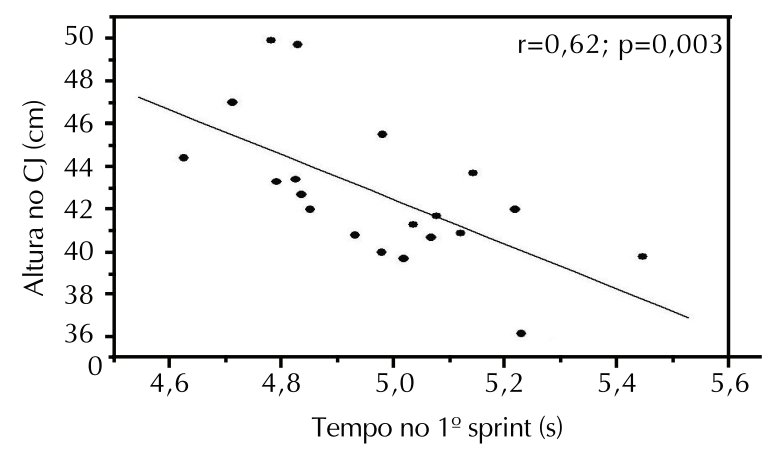

Figura 3 - Relação entre a altura obtida no CJ antes da realização dos sprints e o tempo no primeiro sprint.

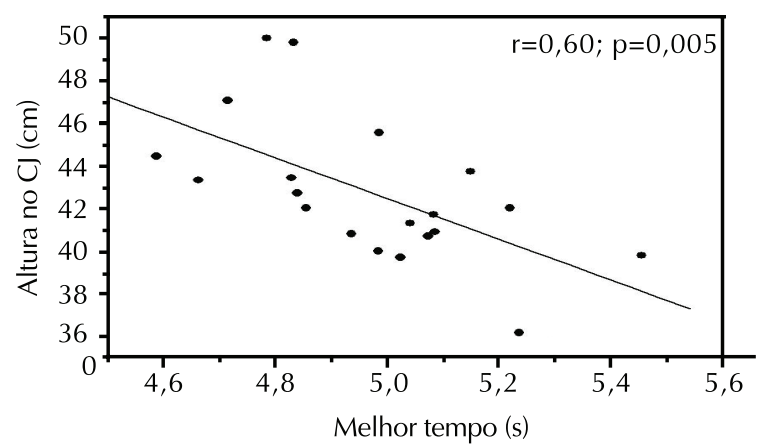

Figura 4 - Relação entre a altura obtida no CJ antes da realização dos sprints e o melhor tempo nos sprints.

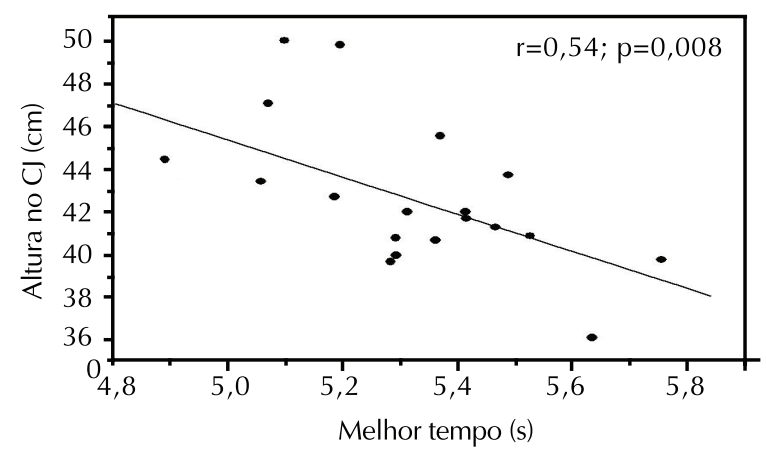

Figura 5 - Relação entre a altura obtida no CJ antes da realização dos sprints e o tempo médio nos sprints.

\section{DISCUSSÃO}

Os principais achados deste estudo indicam que os futebolistas analisados foram capazes de manter os níveis de potência muscular após a realização de sucessivos sprints, além de verificar-se a interdependência entre potência muscular e a CSR.

Conforme hipótese formulada no presente estudo, esperava-se uma redução no desempenho obtido no CJ após a realização dos sprints repetidos. Isso seria justificado em função da elevada solicitação da glicólise no fornecimento energético durante os sprints ${ }^{12}$, tendo em vista a limitação dos estoques de fosfocreatina para suprir a demanda até o final do exercício $^{10}$. A contribuição da via glicolítica pode ser observada, neste estudo, pela concentração de lactato $\left(10,12 \pm 1,48 \mathrm{mmol} \cdot \mathrm{L}^{-1}\right)$ obtida 5 min após os sprints repetidos.

Em estudo prévio ${ }^{16}$, registraram-se valores médios de pico de lactato de $14,23 \pm 2,68 \mathrm{mmol}^{-\mathrm{L}^{-1}}$, chegando a valores máximos de até $19 \mathrm{mmol} \cdot \mathrm{L}^{-1}$ após a realização do RAST test. Nestas situações consideradas láticas, está relatado que há grande formação de íons $\mathrm{H}^{+}$, ocorrendo redução no $\mathrm{pH}$ intra-muscular, o que irá limitar a atividade glicolítica ${ }^{11,12}$. Tem sido sugerido que esse quadro de acidose pode provocar um quadro de fadiga muscular, ocasionando prejuízos no mecanismo contrátil das fibras músculoesqueléticas ${ }^{12}$, o que prejudicaria a produção de potência. Sabe-se que o processo da fadiga muscular é um complexo fenômeno, ocorrendo em numerosos locais por meio de vários mecanismos, que incluem o sistema nervoso central, mas parece que a mesma atua predominantemente por meio de mecanismos localizados dentro do músculo esquelético ${ }^{17}$.

As investigações realizadas nos últimos anos identificaram a importância dos distúrbios metabólicos na fadiga muscular. Conforme revisão elaborada por Bertuzzi et al. ${ }^{12}$, as alterações no $\mathrm{pH}$ resultantes do acúmulo de $\mathrm{H}^{+}$teriam participação 
na inibição da liberação de $\mathrm{Ca}^{+}$, no aumento do $\mathrm{Ca}^{+}$livre, na inibição do motoneurônio, no tempo de transição do estado de ligação das pontes cruzadas de forte para fraca e na inibição das enzimas associadas à glicogenólise e à glicólise. Portanto, $\mathrm{o} \mathrm{pH}$ intracelular diminui de forma progressiva com o aumento da duração do exercício intenso, seguido, supostamente, pela redução da capacidade de gerar tensão a partir do valor de $\mathrm{pH}$ em, aproximadamente $6,8^{18}$.

Presume-se que um fator que possa explicar a manutenção dos níveis de potência após os sprints, verificado no presente estudo, é a capacidade de tamponamento da acidez muscular provocada pela presença elevada dos íons $\mathrm{H}^{+}$, considerada um importante atributo para a $\mathrm{CSR}^{5}$. O músculo esquelético tem a capacidade de controlar as mudanças no $\mathrm{pH}$ por meio do uso de vários mecanismos, tais como o processo químico com bicarbonato, fosfatos e proteínas e hemoglobinas presentes nas células sanguíneas ${ }^{19}$. De acordo com estes autores ${ }^{19}$, estas adaptações parecem ser obtidas com o treinamento de alta intensidade, que está presente nos treinamentos dos jogadores de futebol.

Outro importante mecanismo de controle do pH é a remoção e transporte dos subprodutos do metabolismo como o lactato e o $\mathrm{H}^{+}$. Existem, descritos na literatura, 14 isoformas de transportadores de monocarboxilatos (MCTs), proteínas de membrana que transportam tais substâncias, sendo duas delas (MCT1 e MCT4) encontrados no músculo esquelético $^{20}$. A concentração de MCT1 parece estar relacionada positivamente com a velocidade de remoção de lactato 1-min, após exercício supramáximo (10 ciclos máximos de pedalada) e de modo negativo com um índice de fadiga obtido após o teste ${ }^{21}$.

Resultados de diversas investigações apresentadas por Frollini et al..$^{20}$ mostram que o treinamento de endurance pode aumentar a concentração de MCT1 muscular, ao passo que o treinamento intervalado de alta intensidade pode induzir a aumentos tanto no MCT1 como no MCT4, além do aumento do fluxo sanguíneo no músculo esquelético. Tais alterações nos MCTs contribuem para a remoção de lactato e regulação iônica, fatores importantes para o controle do $\mathrm{pH}$ e consequentemente, na manutenção da produção de força muscular ${ }^{12,21}$.

Em relação à capacidade de realização dos sprints repetidos, mensurada no RAST test, verificou-se um decréscimo da performance dos jogadores no decorrer dos 6 sprints. Tal fato pode ser explicado pela redução do fornecimento energético via sistema anaeróbio alático que, apesar de responder imediatamente à demanda energética exigida durante os movimentos de alta intensidade, é limitado em sua capacidade ${ }^{11}$.

No entanto, verificou-se uma capacidade de manutenção do tempo entre o $5^{\circ}$ e $6^{\circ}$ sprint. Apesar da predominância anaeróbia na CSR, o esforço final pode ser mantido graças ao aumento da participação do sistema aeróbio, que parece responder rapidamente à demanda energética ao final de exercícios intensos ${ }^{22}$. Isso pode ser observado por Bogdanis et al. ${ }^{10}$, ao submeterem indivíduos a realizar dois sprints máximos de 20 e 30 s em cicloergômetro, com dois minutos de recuperação entre os mesmos. Os autores verificaram que a potência média não sofreu grande redução ao final do $2^{\circ}$ sprint, sendo observado um aumento da contribuição aeróbia para a formação de ATP (13 para $27 \%$ do primeiro para o $2^{\circ}$ sprint). Isso demonstra a importância do sistema oxidativo para a recuperação energética em atividades intermitentes de alta intensidade.

Outro estudo que sustenta esta hipótese foi realizado por Gaitanos et al. ${ }^{23}$, no qual oito sujeitos realizaram 10 sprints de $6 \mathrm{~s}$ em um cicloergômetro, com 30 s de intervalo de recuperação. Foram verificadas reduções significativas da contribuição glicolítica no fornecimento energético no $10^{\circ}$ sprint em relação ao primeiro. Desta forma, os autores postulam que, durante os últimos sprints, a glicólise é inibida, sendo o ATP regenerado pelas fontes de $\mathrm{PCr}$, restauradas, parcialmente, no intervalo, e pelo aumento da participação do metabolismo aeróbio.

A contribuição aeróbia na CSR foi também evidenciada na correlação existente entre indicadores de capacidade aeróbia como o OBLA (onset blood lactate accumulation) e potência aeróbia como a $\mathrm{IVO}_{2} \max$ (intensidade relativa ao $\mathrm{VO}_{2} \max$ ) com o TM obtido em um teste de sprints repetidos realizados por jogadores de futebol ${ }^{24}$. $\mathrm{O} \mathrm{VO}_{2}$ max é outro índice aeróbio que está correlacionado moderadamente com o tempo total em sprint repetidos $(8 \times 40$ $\mathrm{m})^{25}$. Estes dados sugerem que o condicionamento aeróbio pode contribuir para a CSR ou para outros aspectos que interferem na performance, durante uma partida, devido à adaptações orgânicas como o aumento do fluxo sanguíneo muscular, dos transportadores de lactato, o aumento da capacidade de regulação iônica, a melhora da eficiência do retículo sarcoplasmático e a maior capacidade de transportar $\mathrm{H}^{+26}$.

A CSR foi analisada, também, por meio de um índice de fadiga (IF) obtido durante a realização dos sprints repetidos no RAST test. Os jogadores ava- 
liados neste estudo apresentaram para este índice o valor médio de $6,79 \pm 1,41 \%$. O IF abaixo de 10 $\%$ indica a capacidade do atleta em manter a performance anaeróbia e, possivelmente, não estaria sofrendo com os efeitos deletérios da fadiga, sendo que valores acima de $10 \%$ apontam que o atleta precisa melhorar sua tolerância aos esforços anaeróbios intermitentes ${ }^{15}$. Tendo em vista os valores apresentados pelos atletas avaliados neste estudo, pode-se sugerir que os mesmos foram capazes de manter a performance após a realização dos sprints, com pouco efeito deletério do possível quadro de fadiga muscular. Esse mecanismo pode explicar e ratificar o fato de não ter ocorrido diminuição significativa de potência muscular entre $\mathrm{Ha}$ e $\mathrm{Hd}$.

As correlações obtidas entre o desempenho nos saltos verticais (Ha) com T1, MT e TM indicam que a realização de um sprint de $35 \mathrm{~m}$, em máxima velocidade, assim como a capacidade em realizar sucessivos sprints em alta intensidade é dependente dos níveis de potência muscular dos jogadores. Tendo em vista que o desempenho nos saltos com contra-movimento, a exemplo do CJ, é condicionada pela ocorrência do ciclo alongamento-encurtamento $(\mathrm{CAE})^{27}$, pode-se dizer que a performance nos sprints é dependente de aspectos neuromusculares relativos ao aproveitamento de energia elástica acumulada durante o CAE. A eficiência deste mecanismo está em grande parte ligada ao nível de stiffness e elasticidade do conjunto músculo-tendíneo, considerados fatores importantes para a performance na ação de $\operatorname{correr}^{28,29}$.

Esses resultados supracitados corroboram investigações realizadas, nas quais foram observadas relações do desempenho em sprints curtos de $5 \mathrm{~m}^{8}$ e de 10 e $30 \mathrm{~m}^{2} \mathrm{com}$ a potência obtida no SJ, assim como do desempenho em sprints de 9,1 e 18,3 $\mathrm{m}^{30} \mathrm{e}$ de $30 \mathrm{~m}^{9} \mathrm{com}$ a altura obtida no CMJ. Tais dados indicam a que a potência muscular associada à capacidade de recrutamento neural e aproveitamento de energia elástica são fatores que podem ser determinantes da CSR.

Não foi verificada correlação significativa entre o desempenho no sexto sprint (T6) e a potência muscular pós RAST test $(\mathrm{Hd})$. Uma possível correlação negativa poderia ser esperada, pois sugere-se que quanto maior o decréscimo de performance em T6, maiores poderiam ser os níveis de fadiga neuromuscular, o que ocasionaria um baixo desempenho nos saltos realizados pós sprints. No entanto, o fato dos jogadores terem apresentado um baixo índice de fadiga $(6,79 \%)$ pode ter contribuído para este fato não ter ocorrido.

\section{CONCLUSÃO}

Os jogadores analisados não apresentaram redução significativa nos níveis de potência após a realização dos sprints repetidos, o que demonstra que a acidose gerada após os estímulos láticos, possivelmente, não tenha comprometido o mecanismo contrátil e consequentemente, os níveis de potência muscular. Em relação à CSR, houve um decréscimo do desempenho do primeiro até o quinto sprint, exceto do quinto para o sexto, indicando a depleção das fontes anaeróbias ao longo dos sprints e uma possível sustentação ao final do esforço pelo aumento da contribuição do metabolismo aeróbio. A partir das correlações obtidas neste estudo, pode-se concluir que a potência muscular é determinante na capacidade dos jogadores realizarem um sprint máximo, assim como a realização de sucessivos sprints (CSR).

\section{REFERÊNCIAS BIBLIOGRÁFICAS}

1. Komi PV. Stretch-shortening cycle: a powerful model to study normal and fatigued muscle. J Biomech 2000;33(10):1197-206

2. Wisloff U, Castagna C, Helgerud J, Jones R, Hoff J. Strong correlation of maximal squat strength with sprint performance and vertical jump height in elite soccer players. Br J Sports Med 2004;38(3):285-8.

3. Hoff J, Helgerud J. Endurance and strength training for soccer players: physiological considerations. Sports Med 2004;34(3):165-80.

4. Impellizzeri FM, Rampinini E, Castagna C, Bishop D, Ferrari Bravo D, Tibaudi A, et al. U. Validity of a repeated-sprint test for football. Int J Sports Med 2008;29(11):899-905.

5. Bishop D, Edge J, Goodman C. Muscle buffer capacity and aerobic fitness are associated with repeated-sprint ability in women. Eur J Appl Physiol 2004;92(4-5):540-7.

6. Impellizzeri FM, Rampinini E, Marcora SM. Physiological assessment of aerobic training in soccer. J Sports Sci 2005;23(6):583-592.

7. Spencer M, Bishop D, Dawson B, Goodman C. Physiological and metabolic responses of repeated-sprint activities. Sport Med 2005;35(12):1025-44.

8. Sleivert G, Taingahue EM. The relationship between maximal jump-squat power and sprint acceleration in athletes. Eur J Appl Physiol 2004;91(1):46-52.

9. Hennessy L, Kilty J. Relationship of the stretch-shortening cycle to sprint performance in trained female athletes. J Strength Cond Res 2001;15(3):326-31.

10. Bogdanis GC, Nevill ME, Boobis, IH. Contribution of phosphocreatine and aerobic metabolism to energy supply during repeated sprint exercise. J Appl Physiol 1996;80(3):876-84. 
11. Gastin PB. Energy system interaction and relative contribution during maximal exercise. Sports Med 2001;31(10):725-41.

12. Bertuzzi RCM, Lima Silva AE, Abad CCC, Pires FO. Metabolismo do lactato: uma revisão sobre a bioenergética e a fadiga muscular. Rev Bras Cineantropom Desempenho Hum 2009;11(2): 226-34.

13. Philp A, MacDonald AL, Watt PW. Lactate - a signal coordinating cell and systemic function. J Exp Biol 2005; 208(24):4561-75.

14. Bosco C. Strength assessment with the Bosco's test. Italian Society of Sport Science: Rome; 1999.

15. Zacharogiannis E, Paradisis G, Tziortzis S. An evaluation of tests of anaerobic power and capacity. Med Sci Sports Exerc 2004;Supp 36(5):116.

16. Zagatto AM, Beck WR, Gobatto CA. Validity of the running anaerobic sprint test for assessing anaerobic power and predicting short-distance performances. J Strength Cond Res 2009; 23(6):1820-7.

17. Leppik JA, Aughey RJ, Medved I, Fairweather I, Carey MF, McKenna MJ. Prolonged exercise to fatigue in humans impairs skeletal muscle $\mathrm{Na}+\mathrm{K}+$ ATPase activity, sarcoplasmic reticulum $\mathrm{Ca} 2+$ release, and $\mathrm{Ca} 2+$ uptake. J Appl Physiol 2004;97(4):1414-23.

18. Cairns SP. Lactic acid and exercise performance: culprit or friend? Sports Med 2006;36(4):279-91.

19. Ross A, Leveritt M, Riek S. Neural influences on sprint running training: adaptations and acute responses. Sports Med 2001;31(6):409-25.

20. Frollini AB, Dias R, Prestes J, Baganha RJ, Cereja DMP, Gomes LPR et al. Exercício físico e regulação do lactato: papel dos transportadores de monocarboxilato (proteínas MCT). Rev Educ Fís/UEM 2008;19(3):453-63.

21. Thomas C, Perrey S, Lambert K, Hugon G, Mornet D, Mercier J. Monocarboxylate transporters, blood lactate removal after supramaximal exercise, and fatigue indexes in humans. J Appl Physiol 2005;98(3):804-9.

22. Duffield R, Dawson B, Goodman C. Energy system contribution to 400 - and 800 -metre track running. J Sports Sci 2005;23(3):299-307.

23. Gaitanos GC, Williams C, Boobis LH, Brooks S. Human muscle metabolism during intermittent maximal exercise. J Appl Physiol 1993;75(2):712-9.
24. Fernandes da Silva, J, Guglielmo, LGA, Bishop, D. Relationship between different measures of aerobic fitness and repeated-sprint ability in elite soccer players. J Strength Cond Res 2010; (in press).

25. Aziz AR, Chia M, Teh KC. The relationship between maximal oxygen uptake and repeated sprint performance indices in field hockey and soccer players. J Sports Med Phys Fitness 200;40(3):195-200.

26. Tomlin DL, Wenger HA. The relationships between aerobic fitness, power maintenance and oxygen consumption during intense intermittent exercise. J Sci Med Sport 2002;5(3):194-203.

27. Bosco C, Tarkka I, Komi PV. Effect of energy elastic and mioeletrical potentiation of triceps surae during stretch-shortening cycle exercise. Int J Sports Med 1982;3(3):137-40.

28. Kubo K, Kanehisa H, Kawakami Y, Fukunaga T. Elasticity of tendon structures of the lower limbs in sprinters. Acta Physiol Scand 2000;168(2):327-35.

29. Kubo K, Morimoto M, Komuro T, Tsunoda N, Kanehisa $\mathrm{H}$, Fukunaga T. Influences of tendon stiffness, joint stiffness, and electromyographic activity on jump performances using single joint. Eur J Appl Physiol 2006;99(3):235-43

30. Vescovi JD, McGuigan MR. Relationships between sprinting, agility, and jump ability in female athletes. J Sports Sci 2008;26(1):97-107.
Endereço para correspondência
Saray Giovana dos Santos
Universidade Federal de Santa Catarina
Centro de Desportos
Campus Universitário - Trindade
Caixa postal 476
CEP - 88040-900 Florianópolis, SC. Brasil
E-mail: saray@cds.ufsc.br 